\title{
Better Security and Privacy for Web Browsers: a Survey of Techniques, and a New Implementation
}

\author{
Willem De Groef, Dominique Devriese, and Frank Piessens \\ IBBT-Distrinet, Katholieke Universiteit Leuven, Belgium, \\ firstname.lastname@cs. kuleuven.be
}

\begin{abstract}
The web browser is one of the most security critical software components today. It is used to interact with a variety of important applications and services, including social networking services, e-mail services, and e-commerce and e-health applications. But the same browser is also used to visit less trustworthy sites, and it is unreasonable to make it the end-user's responsibility to "browse safely". So it is an important design goal for a browser to provide adequate privacy and security guarantees, and to make sure that potentially malicious content from one web site can not compromise the browser, violate the user's privacy, or interfere with other web sites that the user interacts with.

Hence, browser security has been a very active topic of research over the past decade, and many proposals have been made for new browser security techniques or architectures. In the first part of this paper, we provide a survey of some important problems and some proposed solutions. We start with a very broad view on browser security problems, and then zoom in on the issues related to the security of JavaScript scripts on the Web. We discuss three important classes of techniques: fine-grained script access control, capability-secure scripting and information flow security for scripts, focusing on techniques with a solid formal foundation. In the second part of the paper, we describe a novel implementation of one information flow security technique. We discuss how we have implemented the technique of secure multi-execution in the Mozilla Firefox browser, and we report on some preliminary experiments with this implementation.
\end{abstract}

Keywords: privacy, security, web browser, information flow security, secure multi-execution

\section{Introduction}

The world wide web is one of the most influential software infrastructures ever. It is a platform for entertainment, social interaction, e-commerce, e-government, e-health and much more. Technologically, the web platform is defined by a wide variety of complex interacting components. Web browsers, web servers and web protocols have grown in functionality and complexity over many years. This combination of importance and complexity makes the web one of the most interesting challenges from the point of view of security. Both the analysis of 
the web platform for security vulnerabilities, as well as the design of new countermeasures that close vulnerabilities with high assurance and without breaking important web functionalities, can benefit substantially from the use of formal models and techniques [1,6,14].

This paper will zoom in on the security and privacy properties of the web browser. Browser security in itself is a broad field, and encompasses at least the following aspects.

Protection against elevation of privilege attacks against the browser. A browser is a complex piece of software with a large network-facing attack surface. Implementation-level vulnerabilities can allow a remote attacker to compromise the browser, and gain user-level or even administrator-level privileges on the user's computer. A popular class of attacks in this category are drive-bydownloads [30]: when a user visits a site, the site sends malicious content to the browser that exploits a vulnerability to install a malware binary on the user's computer. A recent paper by Provos et al. [29] provides convincing evidence of the pervasiveness of this class of attacks.

Protection against cross-domain attacks. A single browser often interacts with several websites at the same time. A user may have a social networking session open in one tab, a banking session in another tab, and a search page in a third tab. If one of the sites that the browser is interacting with is malicious, that site can try to launch attacks against the other open sites. An important browser vulnerability in this respect is that the browser will automatically add authentication and session information to any outgoing request towards sites that the browser has established a session with. In a cross-site-request-forgery (CSRF) attack [4], the malicious site will return content that will in turn trigger a browser request to one of the other sites that the browser is interacting with. The browser will add authentication and session information automatically, and the attacker generated request will be processed with the user's privileges. Protection against CSRF attacks can be done server-side [4] or browser-side $[12,19]$, and an important challenge in securing the browser-side is in doing this while remaining compatible with other common, non-malicious cross-domain scenarios such as third-party payment and single sign-on [13].

Containment of malicious web scripts. Browsers support active content through JavaScript and popular browser extensions such as Flash and Silverlight. This brings many of the security and privacy risks associated with mobile code to the browser. Malicious scripts can launch cross-domain attacks as discussed in the previous paragraph, for instance by posting a form to another domain. But scripts can also attack the domain to which they belong: there are several ways in which malicious scripts can find their way into web pages. A crosssite scripting vulnerability in the server for instance enables attackers to inject scripts. And many web sites voluntarily include third-party scripts, for instance advertisements from third-party ad-providers are often implemented as scripts that run within the same origin as the including page. Such scripts can then 
violate the integrity of the web page, or leak any user private data on the web page to the attacker.

Besides the three aspects of browser security discussed above, there are many others. Browsers implement cryptographic mechanisms that should satisfy stringent security requirements. Also, browsers can help address social engineering attacks through secure user interface design, or by detecting potentially dangerous actions and warning the user. Browser security is a very broad and interdisciplinary problem, and we can not discuss all aspects of it in a single paper.

For the rest of this paper, we will focus on the problem of dealing with the containment of malicious web scripts. In the next section, we study the problems related to web script security in more detail. Next, in Section 3, we survey three important categories of security techniques that address script security and privacy problems, with an emphasis on approaches that have a strong formal foundation.

Then we come to the second part of the paper, where we present and evaluate a novel implementation of one of these web security techniques. Section 4 briefly recapitulates the technique of secure multi-execution, and Section 5 describes how we implemented this technique in a real browser. Section 6 reports on some preliminary experiments we performed with that implementation, and finally Section 7 concludes the paper.

\section{Web script security: problems}

Modern Internet applications combine both HTML and JavaScript code (also called scripts). These scripts can be part of the HTML page itself (inline scripts), or can be included by specifying in the HTML page the URL where the script can be found. Such remote scripts can be hosted on the same server as the HTML page including them, but scripts can also be included from any other reachable third-party server. When a script is included (inline or remotely) in a web page, it has access to all information in that web page, as well as to potentially sensitive metadata (e.g. document. cookie). Scripts also have access to a number of browser API's, for example an API to send (restricted forms of) HTTP requests.

Current browsers address web script security through a heterogeneous collection of security controls collectively known as the same-origin policy $[32,43]$. An origin is a (protocol, domain name,port) triple, and restrictions are imposed on how code belonging to one origin can interact with data from another origin. For the purpose of enforcing the same-origin policy, the origin of a script is not the origin from which the script is downloaded, but the origin of the HTML page that includes the script. In other words, if a web page author includes a remote third-party script, the author effectively grants that third party script the full set of the web page's privileges, including access to all information in it. 
The same-origin policy provides some basic protection against malicious web scripts, but it has also been widely criticized on the following grounds.

First, the same-origin policy is implemented inconsistently in current browsers [37], it is ambiguous and imprecise [6], and it fails to provide adequate protection for resources belonging to the user rather than to some origin [37]. This is largely due to the fact that the same-origin policy has evolved in an ad-hoc way as new browser features and functionality was introduced over the years.

Second, there are some important vulnerabilities in the same-origin policy with respect to information leakage. Through the browser API's available to them, scripts can effectively transmit information to any server on the internet [18]. For instance, scripts can ask the browser to load an image from a scriptspecified URL, and can encode arbitrary information in that URL.

Third, as discussed above, the same-origin policy does not distinguish between scripts loaded from different origins: it is the origin of the HTML page including the scripts that is taken into account for access control. This makes it non-trivial to provide security guarantees for mashups: web applications that combine code and data from multiple sources $[11,21,23]$. It also makes it hard to securely support third-party widgets or apps through script inclusion. If a social networking site wants to support third-party JavaScript apps through remote script inclusion, the same-origin policy provides no protection and additional security measures will be necessary.

Many authors $[5,16,18,29,37,38]$ provide evidence of the shortcomings of the same-origin policy. We discuss some important example scenarios taken from these papers.

Cookie stealing. A malicious script can access and leak cookie data to the attacker. Since cookies are the most common mechanism for implementing sessions in web applications, cookie stealing can enable the attacker to take over the user session. One can argue, that this issue can be fixed by preventing JavaScript to see the cookie data [27]. This will however break scenario's where the content of the cookie does matter, e.g. because it contains some user-defined settings. A better solution is to prevent leaking the cookie contents.

Behaviour tracking. It is relatively common practice for web sites to gather details of how users interact with web pages [16, §5]. A web site can track mouse movement, scrolling behaviour, information about what text was selected and copied to the clipboard, and so forth by attaching special handlers to all interesting events (e.g. onmouseover when the user goes over on object with his mouse). Browser side protection against such behaviour tracking is non-trivial. Simply denying the installation of event handlers will break many legitimate web pages. Again, a better solution is to allow scripts access to these events, but to prevent the script from leaking this information.

Leaking of user private data. The same-origin policy only addresses protection between origins. Information in the browser that should 
be private to the user is not protected by the same-origin policy. This makes it impossible to implement scenarios where scripts get access to user private data but are prevented from sending this data back to the server. Such user private data could include for instance clipboard data or geolocation information [37]. It could also include application-specific data, for instance in a tax-calculation service where the application provider only offers the necessary scripts to calculate the tax value, based on values entered by the user, but where the information entered by the user is not intended to leak back to the server [5, §2].

Malicious advertisements. Third-party advertisements are commonly implemented through script-inclusion [29, §5.2]. Moreover, ad-providers will often rent out advertisement space to other parties, giving a wide range of stakeholders the opportunity to include scripts. There are several documented incidents [38, §1] of advertisements abusing the privileges they get through script inclusion, and there is even strong evidence of the fact that advertisement scripts are an important vehicle for malware propagation [29].

We can summarize by stating that the same-origin policy used in current browsers is too coarse and even fundamentally unable to protect users against privacy-violating scripts.

\section{Web script security: solutions}

Many proposals for improving web script security have been studied. They can roughly be classified in three categories.

Supporting fine-grained access control on scripts. The basic idea underlying this first class of approaches is to give authors of web pages more control over what included scripts can do. Instead of giving all included scripts full privileges, the author of a web page can specify an access control policy that will then be enforced on scripts included in the page.

Many variations of this approach have been described, that differ in the kinds of policies that can be expressed, and in the implementation technique used to enforce the policy.

Two important implementation techniques have been proposed. ConScript [24] and WebJail [39] enforce policies by implementing a reference monitor in the script execution engine in the browser. BrowserShield [31] and Self-protecting JavaScript [28] enforce policies by rewriting the JavaScript code, essentially inlining a reference monitor in the code. A key advantage of the inlining based approaches is that they do not require browser modifications. An important advantage of building the monitor into the execution engine is that it is relatively easy to make sure that the reference monitor is completely mediating, i.e. that it sees all security relevant actions of the script. For inlining based approaches, this is hard because of the complexities of the JavaScript language. 
With respect to the policies supported, the various proposed systems differ both in the security-relevant events that the policies can talk about; for instance, some systems only regulate access to invocations of native methods [28], others can monitor all JavaScript function invocations [24]. They also vary in the expressivity of the policy language used; some systems expect policies to be written in JavaScript too $[24,28]$ whereas others advocate the use of simpler but less expressive policy languages [39].

Capability secure scripting. Approaches based on capability secure scripting [22] bring the ideas of the object-capability model [26] to web scripts. In this language-based approach to security, the scripting language should be capability secure. Essentially, this means that scripts can only get access to (call methods on) objects that they created or that were explicitly handed to them. ${ }^{1}$ If we assume that all security-relevant API's are implemented as methods of preexisting objects, then this constraint implies that scripts will only get access to that part of the API that is explicitly handed to them. A web page author can get fine-grained control over what dynamically loaded scripts can do, by carefully considering what objects to pass to these scripts.

An important advantage of capability secure scripting is that it offers a powerful foundation. It is relatively straightforward to build fine-grained access control on top of a capability secure scripting system: the reference monitor can be implemented as a wrapper around the object that implements the API to which access needs to be controlled. It is also straightforward to support strict isolation between different scripts on the same page: the integrator just needs to make sure that the objects handed to the different scripts are disjoint. Controlled collaboration between scripts can be achieved by passing them both a reference to an object that implements the desired collaboration protocol. A disadvantage of this approach is that a great deal of responsibility lies with the programmer implementing the API. Essentially, the programmer determines the policy that is enforced, and it is easy to make programming bugs that break the desired security guarantees.

The Caja system [25] is a relatively mature implementation of this approach for JavaScript. Since JavaScript is not a capability-secure language, Caja achieves capability security through program rewriting: programs are rewritten to a subset of JavaScript that can be shown to be capability secure [22].

Information flow security for web scripts. A third class of approaches to script security focuses on controlling how information can propagate through scripts. It applies the wide body of research on information flow security [35] to web scripts. One specifies a policy for a web application by labeling all inputs and outputs to the application with a security label. These labels represent a confidentiality level (or dually an integrity level), and they are partially ordered where one label is above another label if it represents a higher level

\footnotetext{
${ }^{1}$ This is an oversimplification, for a precise formal definition, we refer the reader to Maffeis et al. [22].
} 
of confidentiality (or dually a lower level of integrity). One then tries to enforce that information only flows upward through the program; there should be no downward flows from more confidential inputs to less confidential outputs (or dually from less reliable inputs to more reliable outputs). This is often formalised as a property called non-interference; a deterministic program is non-interferent if there are no two runs of the program with the inputs identical up to a level $l$ such that the program has different outputs at a level below $l$.

While there has been a substantial body of research on information flow security over the past decades, the JavaScript language, and the web context bring significant additional challenges, including for instance dealing with the dynamic nature of JavaScript, and dealing with information flows through the DOM API that the browsers present to scripts $[2,33,34]$.

Again, there has been a wide variety of approaches in this category. They differ on the enforcement mechanism used, and on the security lattices they consider. With respect to enforcement, there are static approaches [10], runtime monitoring based approaches $[9,34]$ and multi-execution based approaches $[5,15,38]$. With respect to the policies considered, some authors focus specifically on providing information flow guarantees for mashup scenarios [21,23] whereas others specifically aim to provide a generic replacement for the same-origin policy $[5,7]$.

Conclusions. The survey of the various approaches to web script security in this section illustrates the importance of the problem, and the vibrant activity in this research field. The different solutions proposed in the literature each have their own advantages and disadvantages in terms of benefits (security guarantees offered), and costs (performance and/or memory overhead, developer involvement and so forth). It is unlikely that one single technique will emerge that subsumes all the others.

For the remainder of this paper, we will now turn our attention to one specific approach - secure multi-execution [15] - for which several implementation techniques have been proposed. We briefly discuss the existing implementation techniques, and we discuss a new implementation in the Mozilla Firefox browser.

\section{Secure Multi-Execution}

Secure multi-execution (SME) $[8,15]$ is a relatively new, dynamic enforcement mechanism for information flow security. Let us assume for simplicity that there are only two security levels, high $(H)$ for confidential information and low $(L)$ for public information. For this simple security lattice, SME will enforce a relatively strong form of non-interference, taking into account termination and timing behaviour of $\mathrm{I} / \mathrm{O}$. The core idea of this mechanism is to execute the program multiple times (one copy of the program for each security level), and to ensure that (1) outputs of a given level $l$ are only done in the execution at level $l$ (outputs are suppressed in other copies), and (2) inputs at a level $l$ are only done at level 
$l$ (for the other copies above $l$, the values that were input by level $l$ are reused, whereas copies that are not above $l$ are fed a default input value).

It is relatively easy to see that executing a program under the SME regime will guarantee non-interference: the copy that does output at level $L$ only sees inputs of level $L$ and hence the output could not have been influenced by inputs of level $H$.

Here is a simple example of how SME would close an information leak in a privacy violating JavaScript program. Consider the following code:

1 var text $=$ document.getElementById('email-input').value;

2 var url = 'http://example.com/img.jpg' + '?t=' + escape(text);

3 document.getElementById('banner-img'). src $=$ url;

This script (running for instance in the context of a web-based e-mail application) encodes the content of an e-mail in a URL, and then loads an image from that URL by setting the src property of an image node. As a consequence, the e-mail content is leaked to the domain example.com, since the browser will issue a GET request for the constructed URL.

SME will close this leak by executing the script twice, using the SME rules for handling input and output. If we label reading from text fields as a high input, and writing to the src property as a low output, and if we use undefined as the default value for inputs, the low copy of the script will run as follows:

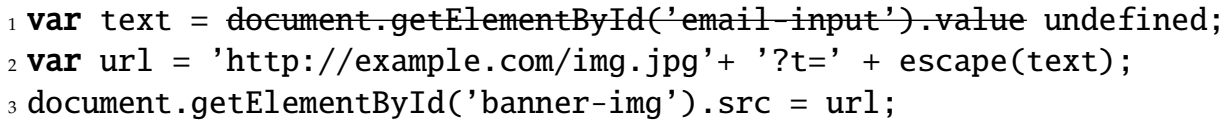

The high copy executes the code as follows:

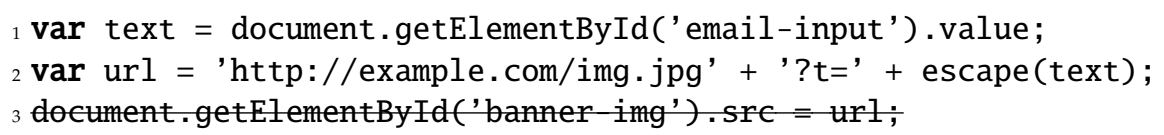

The low copy reads the value undefined, since input from a form text field is classified as high. As a consequence, the URL sent to example.com does not contain the e-mail content. The high copy does have access to the e-mail content, and constructs a URL that encodes this information, but in the high copy the sending of the GET request for the image is suppressed. Hence, SME has rendered the script non-interferent.

Devriese and Piessens [15] have worked out the details of this enforcement mechanism and have proven the key theoretical properties, also for more complex lattices than the two-element lattice considered here, and for timingsensitive variants of non-interference. Kashyap et al. [20] generalize and refine the results of Devriese and Piessens towards a class of enforcement mechanisms that they call the scheduling approach to non-interference. We refer the reader to these two papers for a detailed theoretical treatment.

SME has a relatively high cost in terms of memory and/or performance overhead. But it has also important advantages. SME enforces non-interference with very little developer involvement. SME is also precise in the sense that it 
will have no impact on programs that were non-interferent to start from. This is relatively easy to see: if low outputs did not depend on high inputs to start from, then replacing high inputs with default values will not impact the low outputs. As a consequence, several authors have proposed implementations of SME or similar systems. Capizzi et al. [8] have implemented a technique that is very similar, but independently developed, at the level of operating system processes for the case of two security levels. Bielova et al. [5] have worked out an implementation technique for browsers and implemented it for the Featherweight Firefox [6] browser model. This implementation technique is essentially a generalization of the implementation by Capizzi et al. to more than two security levels. Austin and Flanagan [3] have recently proposed a novel implementation technique based on so-called faceted values that promises to reduce the performance overhead. Jaskelioff and Russo [17] have worked out a library-based implementation for Haskell.

When implementing SME for a browser, an important design choice is how to deal with the Document Object Model API, the API to interact with the web page that the browser exposes to scripts. On the one hand, one can multi-execute the entire browser, and hence DOM API interactions become internal interactions: each SME copy of the browser will have its own copy of the DOM. This is essentially what Bielova et al. and Capizzi et al. do in their implementations. An alternative is to only multi-execute the scripts, and to treat the interactions with the DOM API as inputs and outputs. In the next Section, we report on an implementation that takes this approach.

\section{Implementation}

Our prototype extends the free and open source web browser Mozilla Firefox version 6.0.2 with support for secure multi-execution. This web browser is maintained by the Mozilla Corporation and is currently the second most widely used browser [42].

\subsection{The Mozilla Firefox Architecture}

Mozilla Firefox is built on top of the Mozilla Application Framework, consisting of a collection of platform independent software components also used in other Mozilla applications. The components most relevant for our implementation are the following ones:

XPCOM. XPCOM (Cross Platform Component Object Model) is the implementation of a component model that supports interoperability between components developed in different programming languages. The DOM in Mozilla Firefox is implemented as XPCOM objects.

XPConnect. The XPConnect (Cross Platform Connect) layer provides the technology for XPCOM objects to interoperate with JavaScript; it acts as the glue between JavaScript and the XPCOM components written in $\mathrm{C} / \mathrm{C}++$.

SpiderMonkey. The SpiderMonkey software library is the JavaScript engine of the framework written in $\mathrm{C} / \mathrm{C}++$. 


\subsection{Overview of the implementation}

For simplicity reasons, our prototype only implements serial multi-execution [15, sIII.B], and it only multi-executes web scripts. All interactions of the scripts with the hosting browser (most notably, the DOM interactions) are treated as I/O operations, and should be handled based on the SME I/O rules.

An important design goal was to minimize changes to the Firefox source code. Running multiple instances of the SpiderMonkey engine as was proposed in [15] turned out to be infeasible, because JavaScript is used throughout the implementation of Firefox, and not only for the execution of web scripts. Instead, we opted for modifying the SpiderMonkey component to make it SME aware.

Hence our implementation consists of three important parts: (1) a modified, SME-aware JavaScript engine, (2) an interceptor for all DOM interactions that implements the SME I/O rules, and (3) a modification of event handling that correctly separates the different security levels. We discuss each of these in some more detail in the following subsections. Other parts of the implementation, such as the modification of the web page parser to correctly initiate multiexecution of web scripts are relatively straightforward and are not further discussed.

\subsection{SpiderMonkey}

All execution of JavaScript code in SpiderMonkey is done in a specific context, and information about the context is maintained in the JSContext data structure. JavaScript objects in SpiderMonkey are represented by JSObject data structures. We implement separation between the different security levels by making the view of a JSObject dependant on the security level of the JSContext in which it is accessed (Figure 2 is the view of the objects in Figure 1 when the security level of the context is low $(L)$ ). We achieve this by building on the concept of shapes in the SpiderMonkey implementation.

Shapes are a simple representation of the meta information of properties (e.g. getters, setters or attributes), and SpiderMonkey uses them to optimize the internal usage of properties. We extend the Shape data structure to hold the security level so that whenever a property of a JSObject is looked up, only Shapes with the appropriate security level can be manipulated. We note a Shape as the triple \{security level, property name, type\}. The result is a JSObject that can behave totally different, depending on the security level of the JSContext it is used in, as shown in Figure 1 and Figure 2. Figure 1 shows a snapshot of a small fragment of the JavaScript heap with the shape information that is maintained for each object, and Figure 2 shows what part of the heap would be visible in a low $(L)$ execution context.

Because SpiderMonkey is also used in the browser infrastructure itself (e.g. to generate the GUI) where it is never necessary to multi-execute, there must be a way to completely disable SME. When operating in the disabled mode, SpiderMonkey functions in an identical way as the non-enhanced version. To support this temporarily shutdown of SME, our implementation also extends 
Fig. 1: Extended JSObjects for secure multi-execution

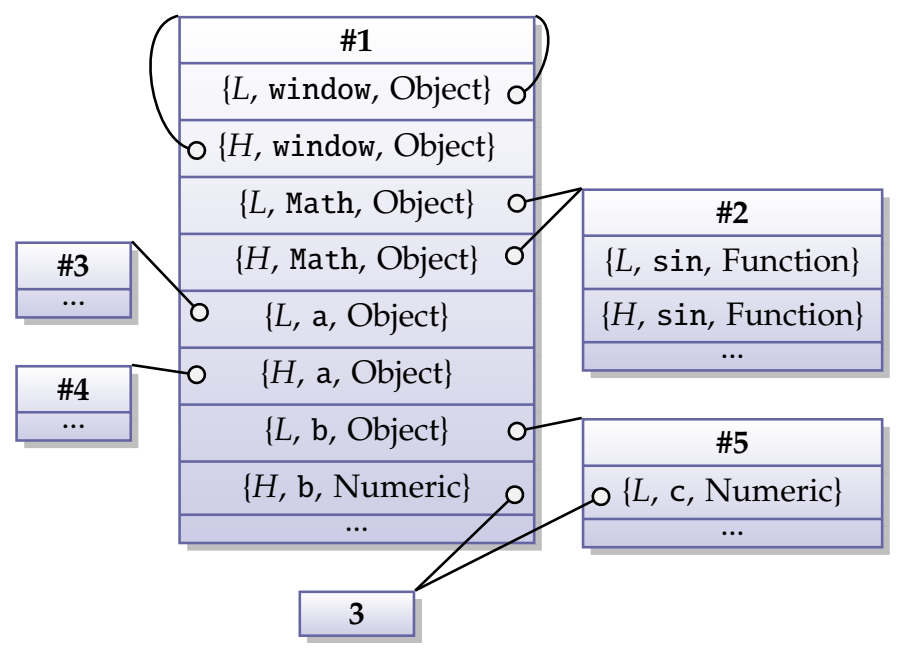

Fig. 2: Extended JSObjects in JSContext with security level $L$

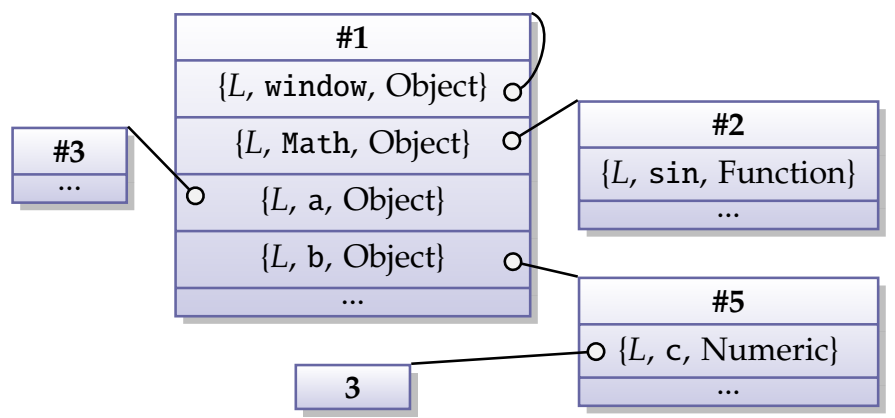

the JSContext data structure with state information related to the execution behaviour of SME. Apart from the disabled mode, a JSContext can be in one of the following two states:

Active. In the active state, a JSContext runs at a specific security level. All actions of a script happen only in that specific security level. In Figure 1, the object \#5 (window.b) was created in a low execution context when SME was active, and hence is only visible on level L. Objects \#3 and \#4 (window.a) were created when SME was active and both levels created a window.a object. Hence, there are two separate objects, one for each level. In the $L$ level, window. a refers to object \#3 ( Figure 2 ) and in the $H$ level window.a refers to object \#4. The active state occurs only when JavaScript programs, 
found while parsing a HTML document, are executed and whenever events are handled.

Initializing. During the initialization of a JSContext (and more specifically during the initialization of the global object \#1 (window) and the standard objects like Math in Figure 1), all the globally defined symbols (i.e. symbols available to every JavaScript program like e.g. Math.sin) have to be introduced in all available security levels to ensure that all possible security levels have access to those standard properties. This can be achieved by multi-executing the functionality to introduce a new property in a JSObject. This is what happens in the initializing state, and this is how object \#2 (window.Math) in Figure 1 ended up being shared between the $H$ and $L$ levels.

The next subsection will discuss an interceptor for DOM interactions in order to implement the SME I/O rules as defined in Section 4.

\subsection{DOM Interactions}

All DOM interactions are handled as $\mathrm{I} / \mathrm{O}$ calls, so we have to implement the SME I/O rules for them. We have to skip DOM interactions that are classified as outputs on all levels except the level at which the output is classified. For input, we consider two cases:

Input without side effects. Several DOM API calls (for instance the reading of document.cookie) are classified as inputs and have no side effects. Executions that are allowed to see the input just proceed as before. For executions with a lower security level than that of the input channel, we simply skip the operation (which would result in an undefined as in the example in Section 4). Alternatively, we can return a specific default value, e.g. the empty string.

Input with side effects. For input with side effects (e.g. reading the response from a prompt call), we have to store the input data in order to reuse it on a later point in time in other executions.

Skipping of DOM API calls can be implemented very generically: We modified the XPConnect layer to intercept all possible DOM API calls and to mediate their execution. Whenever a DOM API call is made from JavaScript (e.g. via document.cookie), this call will first be marshalled in the XPConnect layer. We intercept this action and invoke a skipcall function (see Figure 3). This function will decide, based on the specific call and the security level of the current JSContext, if the corresponding XPCOM call will be executed or not. Whenever the skipCall function returns false, the call in XPConnect returns as it would do under normal circumstances but without passing execution on to the relevant XPCOM component. This effectively results in skipping of the instruction.

Our prototype implementation makes it easy to modify the skipcall function. It is written in plain JavaScript to make it easy to experiment with 
different implementations (that cover different fragments of the DOM API for instance) of skipCall. An example implementation for the example in Section 4 can be found in Figure 3.

Fig. 3: The skipCall function for interposition on DOM API calls

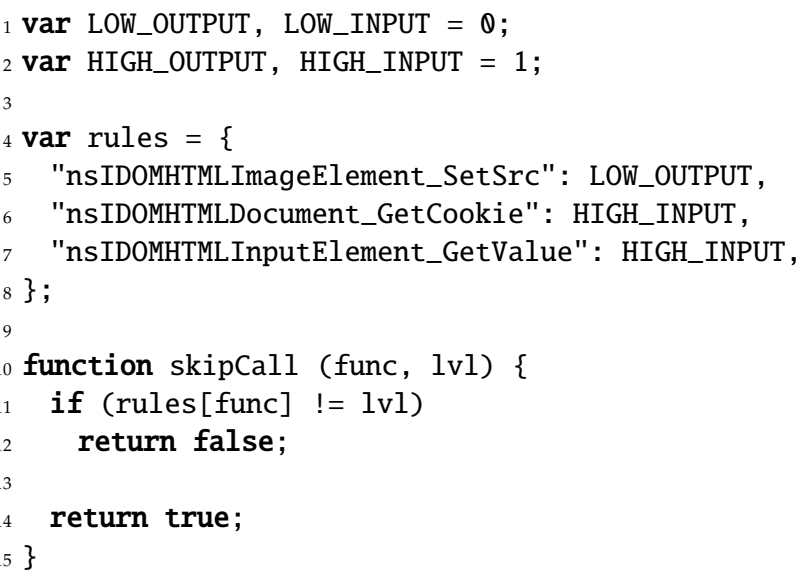

For input with side effects, we augment the JSContext data structure to temporarily save the input data, to allow reuse of the input data on higher security levels. For instance, we modified the implementation of prompt to save the response. Whenever a DOM API call for prompt is made (to read the response) on a higher security level, we act as if the execution is skipped and return the saved value from the current JSContext.

\subsection{Event Handling}

Mozilla Firefox supports DOM events as specified by [36]. From within JavaScript, it is possible to attach event handlers to various objects. Whenever an event occurs, the right event handler will be invoked. This works by looping over all installed event handlers and only invoking event handlers for the corresponding triggered event.

Devriese and Piessens [15] mention that browser callbacks are not formally modelled in their model of SME. They describe a formal encoding of an implementation where "callbacks are executed only in executions at security level $l$ or higher, with $l$ the security level assigned to the relevant event" [15, §VI.A]. Bielova et al. implemented this for the Featherweight Firefox model [5].

Our implementation follows the same idea. Up to now, we have not yet implemented a mechanism to attach security levels to events. Our implementation treats all events as low $(L)$ inputs. Any level can attach event 
handlers to any event. We save the security level of the JSContext in which a handler is installed, together with the event handler itself. When an event fires, all event handlers for that event are executed, but for each handler that is executed the security level of the JSContext is changed, so that the event handler code is executed within the right security level. Once we implement a mechanism to assign security levels to events, this implementation needs a minor change so that it only delivers an event at a given level to handlers with level equal to or above the level of the event.

\section{Preliminary Evaluation}

Our implementation is still incomplete: the current prototype only handles a small fraction of the DOM API, and extending this to the full DOM API is a significant engineering effort. However, the implementation is sufficiently complete to draw some conclusions with respect to performance cost. We report these preliminary conclusions in this Section.

\subsection{Micro Benchmarks}

In their presentation of SME [15], Devriese and Piessens implemented a very simple model browser, and measured the impact on performance and memory overhead. Their measurements showed that for serial multi-execution execution time more or less doubles. For I/O intensive code however, the fact that input values are reused for higher security levels can lead to lower run time overheads (in extreme cases even leading to speedup for the parallel implementation of SME).

The goal of our micro benchmarks is to (i) evaluate the performance cost of our implementation and (ii) verify that the results from Devriese and Piessens (including the lower performance cost for $\mathrm{I} / \mathrm{O}$ intensive programs) also hold for our new implementation.

As a test benchmark for our modified SpiderMonkey, we used the Google Chrome v8 Benchmark suite version 6. This suite was developed to optimize the JavaScript interpreter for Google Chrome. It contains both mathematical tests as data structure efficiency tests. We reused the I/O test from $[15, \S \mathrm{V} . \mathrm{B}]$ to simulate an I/O intensive program, listed in Figure 4. This test uses all available security levels and simulates some form of latency, e.g. network latency. Specifically, we used a value of $10 \mathrm{~ms}$ in our tests.

The results are shown in Figure $5^{2}$. With SME disabled, there is only a negligible impact on performance. This is important, as Firefox will not only execute web scripts in the JavaScript engine. Also a substantial fraction of the Firefox code itself is executed in the JavaScript engine, and for such code the

\footnotetext{
${ }^{2}$ All tests were performed on a machine with an Intel@Core ${ }^{\mathrm{TM}} 2$ Quad CPU Q6600 2.4Ghz and 4GB RAM. For the tests, we restricted the security lattice to a basic two element lattice as defined in Section 4.
} 
Fig. 4: Source code of the I/O intensive simulation benchmark

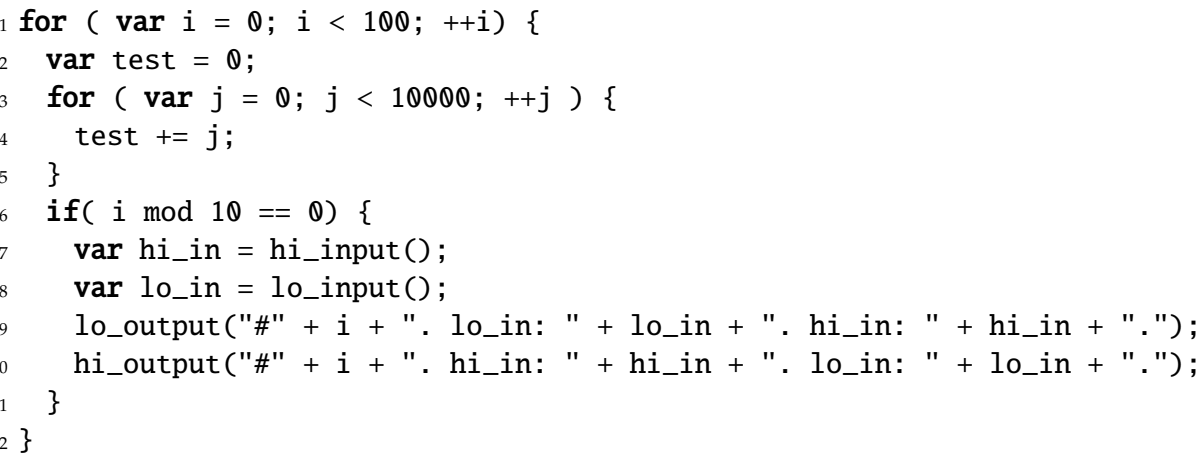

JavaScript engine will run with SME disabled. The micro benchmarks shows that this can be done without impact on performance.

The results confirm our expectations that our prototype more or less doubles execution time when secure multi-execution is active and that the performance cost is substantially smaller for $\mathrm{I} / \mathrm{O}$ intensive code. The results are also in line with those from Devriese and Piessens [15, §V.C].

Fig. 5: Benchmarks for SpiderMonkey with secure multi-execution
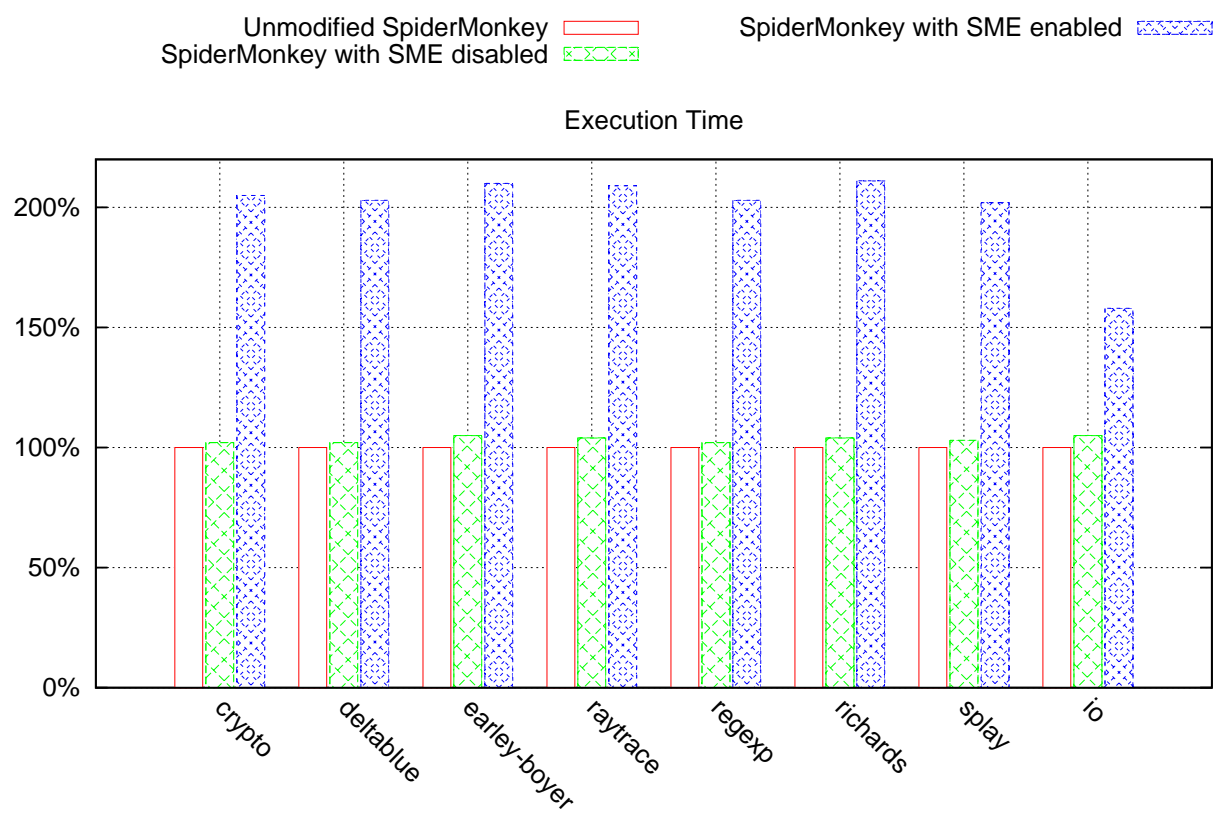


\subsection{User-perceived latency}

Our micro benchmarks show that the overhead of serial SME on the Javascript execution time is large, even for the simple high-low lattice we are using. However, in order to evaluate the cost of applying SME in the browser, the overhead of executing Javascript should be considered as part of the full userperceived latency. Network latency in particular is typically responsible for an important amount of time and may make even an overhead of $100 \%$ on JavaScript execution time acceptable. Unfortunately, our current prototype implementation is insufficiently mature to conduct real-life macro benchmark tests. In this section, we provide some rough estimates of the costs of using serial SME on real websites.

The overhead induced by SME on the total page load time can be approximated as follows:

$$
f(N, R, J)=\frac{N+(1-J) R+2 J R}{N+R}
$$

where $N$ is the network latency for the request, $R$ is the overall time the browser spends to render the page, and $J$ is the fraction of the time that the browser is executing scripts during page rendering. This is an overestimation, as it only takes into account one network roundtrip, whereas page loads often require several roundtrips (for instance to load images and remote scripts) and hence network latency will take up a larger fraction of the total page load time.

Obviously, each of these three parameters can vary widely depending on the type of web page, the geographical location of browser and server, and many other factors. To compute some indicative value of the performance of our implementation in real settings, we make some rough estimates as follows. For network latency $(N)$, basic tests of requests from our department network to the top 5 of the most visited Belgian web sites ${ }^{3}$ yield an estimate of $0.16 s \leq N \leq 0.55$ s.

For total rendering time $(R)$ and fraction of time spent in the JavaScript engine $(J)$, we use some experimental data gathered by Weber [41]. He analyzes the loading of two types of sites, news sites and AJAX sites, measuring the overall loading time, and the amount of time spent in various browser subsystems including the JavaScript subsystem. From Weber's analysis for news sites, we can obtain estimates of $1 s \leq R \leq 3 s$, and $J$ around 0.3 .

Based on these numbers, we can estimate the overhead of serial SME on total page load time between $19 \%$ and $28 \%$. Obviously, this is a very rough estimate, and further experiments are needed to provide more reliable indications of overhead. This is an important avenue for future work.

\section{Conclusions}

Web browsers look more and more like operating systems: they execute programs belonging to different mutually distrusting origins, they implement

\footnotetext{
${ }^{3}$ We used the top 5 websites from http://www.alexa.com/topsites/countries/BE
} 
isolation between these programs, and they manage the resources available to these programs. It is interesting to see that many of the classic approaches to operating system security, such as reference monitors, access control, capability security and information flow security resurface in the context of browser security. Some authors [40] have even argued that browsers should evolve into real operating systems that exclusively manage the resources shared between various web origins.

Given the significant advances in mechanisms for enforcing information flow security, and given that there is empirical evidence that web sites are using web scripts to violate user privacy, it is possible that information flow security will play a more important role in web script security than it has played in operating system security.

This paper has surveyed some of the approaches to deal with security of web scripts recently proposed in the literature, and it has described a novel implementation technique for one of the information flow security enforcement approaches. The main novelty of the proposed implementation technique is that it implements secure multi-execution by only multi-executing the web scripts, treating interaction with the browser API's as I/O. Our hope is that such an implementation technique can reduce the performance cost of secure multi-execution to an acceptable level. There is however significant additional work needed to validate this. In the first place, macro-benchmarks with our implementation should confirm the positive impact on performance overhead. Second, treating DOM interactions as I/O is an approximation that might not be acceptable in all cases. Secure multi-execution of the web scripts alone does not guarantee that the browser as a whole satisfies non-interference. The policy definition should also classify the DOM API in such a way that the script cannot somehow still obtain high information through APIs classified as low. An important question for future work is whether good policies can be defined for the interactions of web scripts with the DOM API such that attack scenarios are blocked but compatibility with non-malicious scenarios is maintained.

Acknowledgments This research is partially funded by the Interuniversity Attraction Poles Programme Belgian State, Belgian Science Policy, by the IWT SPION project, the Research Fund K.U.Leuven and the EU-funded FP7-projects WebSand and NESSoS. Dominique Devriese holds a Ph. D. fellowship of the Research Foundation - Flanders (FWO).

\section{References}

1. Akhawe, D., Barth, A., Lam, P.E., Mitchell, J., Song, D.: Towards a formal foundation of web security. In: Computer Security Foundations Symposium, IEEE. pp. 290-304 (2010)

2. Askarov, A., Sabelfeld, A.: Tight enforcement of information-release policies for dynamic languages. In: CSF. pp. 43-59 (2009)

3. Austin, T., Flanagan, C.: Multiple facets for dynamic information flow. In: POPL (2012) 
4. Barth, A., Jackson, C., Mitchell, J.C.: Robust defenses for cross-site request forgery. In: 15th ACM Conference on Computer and Communications Security (CCS 2008) (2008), http://seclab.stanford.edu/websec/csrf/csrf.pdf

5. Bielova, N., Devriese, D., Massacci, F., Piessens, F.: Reactive non-interference for a browser model. In: Proceedings of the International Conference on Network and System Security (NSS) (September 2011)

6. Bohannon, A., Pierce, B.C.: Featherweight Firefox: Formalizing the core of a web browser. In: WebApps (2010)

7. Bohannon, A., Pierce, B.C., Sjöberg, V., Weirich, S., Zdancewic, S.: Reactive noninterference. In: CCS (2009)

8. Capizzi, R., Longo, A., Venkatakrishnan, V., Sistla, A.: Preventing information leaks through shadow executions. In: ACSAC (2008)

9. Chudnov, A., Naumann, D.A.: Information flow monitor inlining. In: CSF. pp. 200214 (2010)

10. Chugh, R., Meister, J., Jhala, R., Lerner, S.: Staged information flow for Javascript. In: PLDI (2009)

11. De Ryck, P., Decat, M., Desmet, L., Piessens, F., Joosen, W.: Security of web mashups: a survey. In: 15th Nordic Conference in Secure IT Systems (NordSec 2010). Springer (2011), https://lirias . kuleuven.be/handle/123456789/317390

12. De Ryck, P., Desmet, L., Heyman, T., Piessens, F., Joosen, W.: Csfire: Transparent client-side mitigation of malicious cross-domain requests. In: Lecture Notes in Computer Science. vol. 5965, pp. 18-34. Springer Berlin / Heidelberg (February 2010), https://lirias.kuleuven . be/handle/123456789/260893

13. De Ryck, P., Desmet, L., Joosen, W., Piessens, F.: Automatic and precise client-side protection against csrf attacks. In: Lecture Notes in Computer Science. vol. 6879, pp. 100-116. Springer (September 2011), https://lirias.kuleuven.be/handle/ $123456789 / 311551$

14. De Ryck, P., Desmet, L., Philippaerts, P., Piessens, F.: A security analysis of next generation web standards. Tech. rep., European Network and Information Security Agency (ENISA) (July 2011), https://lirias.kuleuven.be/handle/123456789/ 317385

15. Devriese, D., Piessens, F.: Noninterference through secure multi-execution. In: Proceedings of the IEEE Symposium on Security and Privacy. pp. 109-124 (May 2010)

16. Jang, D., Jhala, R., Lerner, S., Shacham, H.: An empirical study of privacy-violating information flows in JavaScript web applications. In: Proceedings of the ACM Conference on Computer and Communications Security (CCS). pp. 270-283. ACM Press (October 2010)

17. Jaskelioff, M., Russo, A.: Secure multi-execution in haskell. In: Proceedings of Andrei Ershov International Conference on Perspectives of System Informatics (PSI'11). Akademgorodok, Novosibirsk, Russia (2011)

18. Johns, M.: On JavaScript Malware and related threats - Web page based attacks revisited. Journal in Computer Virology 4(3), 161 - 178 (August 2008)

19. Johns, M., Winter, J.: RequestRodeo: client side protection against session riding. Proceedings of the OWASP Europe 2006 Conference, refereed papers track, Report CW448 pp. 5-17 (2006)

20. Kashyap, V., Wiedermann, B., Hardekopf, B.: Timing- and termination-sensitive secure information flow: Exploring a new approach. In: Proceedings of the 2011 IEEE Symposium on Security and Privacy. pp. 413-428. SP '11, IEEE Computer Society, Washington, DC, USA (2011), http://dx . doi .org/10 . 1109/SP . 2011. 19 
21. Li, Z., Zhang, K., Wang, X.: Mash-IF: Practical information-flow control within client-side mashups. In: Proceedings of the IEEE/IFIP International Conference on Dependable Systems \& Networks (DSN). pp. 251-260. IEEE (2010)

22. Maffeis, S., Mitchell, J.C., Taly, A.: Object capabilities and isolation of untrusted web applications. In: IEEE Symposium on Security and Privacy. pp. 125-140 (2010)

23. Magazinius, J., Askarov, A., Sabelfeld, A.: A Lattice-based Approach to Mashup Security. In: Proceedings of the ACM Symposium on Information, Computer and Communications Security (ASIACSS). pp. 15-23 (2010)

24. Meyerovich, L., Livshits, B.: ConScript: Specifying and enforcing fine-grained security policies for Javascript in the browser. In: IEEE Symposium on Security and Privacy (May 2010)

25. Miller, M.S., Samuel, M., Laurie, B., Awad, I., Stay, M.: Caja: Safe active content in sanitized javascript. http://google-caja.googlecode.com/files/ caja-spec-2008-01-15.pdf (January 2008)

26. Miller, M.: Robust composition: towards a unified approach to access control and concurrency control. Ph.D. thesis, Johns Hopkins University (2006)

27. Nikiforakis, N., Meert, W., Younan, Y., Johns, M., Joosen, W.: SessionShield: Lightweight protection against session hijacking. In: Proceedings of the International Symposium on Engineering Secure Software and Systems (ESSoS). pp. 87-100 (February 2011)

28. Phung, P.H., Sands, D., Chudnov, A.: Lightweight self-protecting javascript. In: Proceedings of the 4th International Symposium on Information, Computer, and Communications Security. pp. 47-60. ASIACCS '09, ACM (2009), http://doi . acm. org/10.1145/1533057.1533067

29. Provos, N., Mavrommatis, P., Rajab, M.A., Monrose, F.: All your iframes point to us. In: Proceedings of the 17th conference on Security symposium. pp. 1-15. USENIX Association, Berkeley, CA, USA (2008), http://dl.acm.org/citation. cfm?id=1496711. 1496712

30. Provos, N., Mcnamee, D., Mavrommatis, P., Wang, K., Modadugu, N., Inc, G.: The ghost in the browser: Analysis of web-based malware. In: In Usenix Hotbots (2007)

31. Reis, C., Dunagan, J., Wang, H.J., Dubrovsky, O., Esmeir, S.: BrowserShield: vulnerability-driven filtering of dynamic HTML. In: OSDI '06: Proceedings of the 7th symposium on Operating systems design and implementation. pp. 61-74. USENIX Association (2006), http://citeseerx.ist .psu. edu/vi ewdoc/summary?doi=10 . 1 . 1.85 .1661

32. Ruderman, J.: Same origin policy for JavaScript (2010)

33. Russo, A., Sabelfeld, A.: Securing timeout instructions in web applications. In: CSF. pp. 92-106 (2009)

34. Russo, A., Sabelfeld, A., Chudnov, A.: Tracking information flow in dynamic tree structures. In: ESORICS. pp. 86-103 (2009)

35. Sabelfeld, A., Myers, A.C.: Language-based information-flow security. In: JSAC. vol. 21, pp. 5-19 (2003)

36. Schepers, D., Rossi, J.: Document Object Model (DOM) Level 3 Events Specification (2011)

37. Singh, K., Moshchuk, A., Wang, H.J., Lee, W.: On the incoherencies in web browser access control policies. In: Proceedings of the IEEE Symposium on Security and Privacy. pp. 463-478 (2010)

38. Ter Louw, M., Ganesh, K.T., Venkatakrishnan, V.: Adjail: Practical Enforcement of Confidentiality and Integrity Policies on Web Advertisements. In: Proceedings of the USENIX Security Symposium. pp. 24-24 (2010) 
39. Van Acker, S., De Ryck, P., Desmet, L., Piessens, F., Joosen, W.: Webjail: Least-privilege integration of third-party components in web mashups. In: ACSAC (December 2011), https://lirias.kuleuven. be/handle/123456789/316291

40. Wang, H.J., Grier, C., Moshchuk, A., King, S.T., Choudhury, P., Venter, H.: The multi-principal os construction of the gazelle web browser. In: USENIX Security Symposium. pp. 417-432 (2009)

41. Weber, J.: Performance: Profiling how different web sites use browser subsystems. http://blogs.msdn.com/b/ie/archive/2010/08/30/ performance-profiling-how-different-web-sites-use-browser-subsystems . aspx (August 2010)

42. Wikipedia: Usage share of web browsers (October 2011)

43. Zalewski, M.: Browser security handbook. http://code.google.com/p/ browsersec/wiki/Main (2010) 